\title{
Nachtrag zu den Zoocecidien Lothringens
}

von

J. J. Kieffer in Bitsch.

* Die neuen Cecidien werden mit einem Sternchen bezeichnet.

Acer Pseudoplatanus L. und Platanoides L. Dipteroc. Diplosis sp.? Sehr kleine, von einer hellen kreisförmigen Zone umgebene Grübchen auf der unteren Blattfläche. Diese in Lothringen auf $A$. campestris $L$. verbreiteten Missbildungen beobachtete ich nun auch auf den beiden genannten Ahorn-Arten in der Nähe von Schloss La Grange bei Diedenhofen.

* Aira caespitosa L. Dipteroc. Lasioptera calamagrostidis Rbs. Kaum merkliche Halmschwellung oberhalb des zweiten oder dritten Knotens. Die rothen Larven in Mehrzahl zwischen Halm und Blattscheide. Verw. i. E. Wald von La Grange.

Buxus sempervirens L. Phytopt. Phytoptus Canestrinii Nal. Knospen verdickt und abnorm behaart. Häufig in einem Walde bei Sierck.

Calamagrostis epigeios L. Dipteroc. Lasioptera calamagrostidis Rbs. Deformation wie bei Aira. Wald von La Grange.

*Carex contigua Hopp. Dipterocecidium. Dasyneura sp.? Deformation der Fruchtschläuche, welche stark verlängert (etwa $8 \mathrm{~mm}$. lang), am Ende verschmälert und geschlossen sind. Die rothe Larve lebt einzeln in diesen deformirten Schläuchen und hat eine gestielte Gräte; sie gehört in die Gattung Dasyneura. Diese Deformation ist wohl mit der von Hieronymus (1890) und schon früher von H. Loew auf C. muricata beobachteten Missbildung identisch. Dagegen scheint die ebenfalls auf C. muricata lebende Dasyneura muricatae Meade ${ }^{1}$ ) nicht hierher zu gehören; von ihr heisst es näm-

1) Herr Meade hatte die Freundlichkeit mir mehrere Exemplare der von ihm beschriebenen Mücke zu senden. Ich konnte mich also überzengen, dass letztere zur Gattung Dasyneura Rond. (Dichelomyia Rübs ) gehört.

XLII. Heft I u. II. 
lich: „Die befallenen Pflanzen wurden im Juli gesammelt. Zu dieser Zeit ernährte sich die Larve auf Kosten der verkümmerten Blüthentheile und lag in den am Ende offenen und so das Hervorragen der Narben gestattenden Fruchtschläuchen verborgen. Inmitten dieses Säckchens verpuppt sich dieselbe gegen Ende des Herbstes, indem sie sich zuvor einen dünnen papierartigen Cocon spinnt." Bekanntlich hat schon früher Fr. Löw in den Fruchtschläuchen von C. aren aria eine Larve mit stielloser Gräte beobachtet, welche keine Deformation veranlasst. Auch die von Winnertz aus den männlichen Blïthen von C. riparia gezogene Dasyneura ripariae Winn. bewirkt keine Missbildung.

Die Deformation von C. contigua stammt aus dem Illkircher Wald bei Strassburg i. E., wo sie von Herrn Petry im Juni 1892 entdeckt worden ist.

Carex contigua var. umbrosa. Dieselbe Deformation und dieselbe Larve. Ich erhielt sie von Herrn Dr. K neucker, welcher sie im Juli 1892 bei Neureuth in Baden gesammelt hatte.

* Carex Davalliana Sm. Dipterocecidium. Hormomyia sp.? Galle länglich, an beiden Enden verschmälert, etwa $8 \mathrm{~mm}$. lang, glänzend, weisslich oder strohgelb, hart, mit einer oder mehreren Larvenkammern. Einzeln oder zu mehreren an der Stengelbasis. Die grosse weisse Larve unterscheidet sich von den bisher bekannten Arten durch die Gestalt ihrer Gräte. Ich fand dieses Cecidium im Herbar unserer Anstalt, nämlich in Billot's Flora Galliae et Germaniae exsiccata; die Exemplare stammen aus Strassburg i. E., nach der Angabe: "Recueilli dans les fossés de la citadelle de Strasbourg. Juillet 1857."

*Carex divulsa Good. Dipterocecidium. Dasyneura sp.? Deformation der Fruchtschläuche wie auf C. contigua. Auch die Larve ist dieselbe hier wie dort. Dieses ebenso wie folgendes Cecidium wurde bisher nicht im Reichslande beobachtet, ich erhielt beide von Herrn Dr. Kneucker. Hardtwald bei Karlsruhe. Legit. Kneucker, 25. 5. 1891.

*Carex Pairaei Fr. Schultz. Dipterocecidium. Dasyneura sp.? Deformation wie bei voriger Art. Hardtwald bei Karlsruhe. Legit. Kneucker, 2. 7. 1892. Ausserdem sandte mir Herr Dr. Kneucker Exemplare mit der Angabe: „Legit. Döll, 1850. Hardtwald bei Karlsruhe. Döll hielt die Pflanze für C. muricata var." Larve wie auf C. divulsa.

Centaurea Scabiosa L. Hymenopteroc. Aulax Rogenhoferi Wachtl. Geschwollene Akenen, sowie hanfkorngrosse Schwellungen an der Basis der Hüllblätter, einzeln oder zu zwei. Dieselben Pflan- 
zen zeigten auch die von mir beschriebenen fleischigen Verdickungen des Stengelgrundes, ferner auch erbsengrosse Schwellungen auf dem Stiel und der Mittelrippe der Wurzelblätter und der unteren Stengelblätter; letztere werden von Aulax Fitschi Kieff. $\left.{ }^{1}\right)$ erzeugt. Diedenhofen.

*Cerastium alsinoides Lois. Hemipteroc. Aphis cerastii Kalt. Schopfförmige Triebspitzendeformation. Bitsch.

*Cerastium glomeratum Thuill. 1. Hemipteroc. Trioza cerastii H. Lw. Knospenförmige Triebspitzendeformation. Bitsch.

*2. Phytopteroc. Phytoptus cerastii Nal. Blattdeformation. Bitsch.

Chrysanthemum Leucanthemum L. Dipteroc. Contarinia chrysanthemi Kieff. Blüthenköpfe geschlossen bleibend oder nur halb geöffnet und seicht geschwollen. Wenn die Larven in geringer Anzahl vorhanden sind, so ist keine Deformation des Blumenbodens zu bemerken; die einzelnen Scheibenblüthen, an denen die Larven saugen, sind jedoch nicht normal entwickelt und öffnen sich nicht. V. i. E. Bitsch, Bolchen und La Grange.

Corylus Avellana L. Dipteroc. 1. Blattgrübchen. Auch auf C. tubulosa. Bitsch.

2. Kleine, kreisrunde Blattparenchymgallen.* Bitsch.

Dactylis glomerata L. Dipteroc. Mayetiola dactylidis Kieff. Schwellung der Halmbasis; in einer seichten Einsenkung unter den Blattscheiden liegen mehrere weisse Larven, welche sich in einem aus ihrer Larvenhaut dargestellten Tönnchen daselbst verpuppen. Häufig in einem Waldwege bei La Grange.

Eupatorium cannabinum L. Hemipt. Aphis sp.? Blattrandrollung und Triebspitzendeformation. Darin honiggelbe BremiaLarven. Bitsch.

Euphorbia Cyparissias L. Dipteroc. Dasyneura Löwi Mik. Kapselförmige, in eine Spitze ausgezogene Gallen an den Triebspitzen und in den Blüthenständen. Bitsch.

Evonymus europaeus L. Hemipt. Aphis sp.? Blattrandrollung nach unten. Bitsch.

Fraxinus excelsior L. Dipteroc. Kleine, kreisförmige Blattparenchymgallen. Larve nicht beobachtet. La Grange.

*Galeopsis tetrahit L. Dipteroc. Dasyneura galeopsis n. sp. Blüthen geschlossen bleibend und schwach verdickt. Larve weiss, einzeln. V. i. E. An derselben Pflanze waren die Früchte von einer Menge kleiner, weisser Clinodiplosis-Larven bedeckt, während ihre mit Erysiphe überzogenen Blätter citronengelbe Mycodiplosis-Larven trugen. Mit voriger.

$\left.{ }^{1}\right)$ Vgl. Species des Hyménoptères d'Europe et d'Algérie. Les Cynipides. 1897. 
Galium silvestre L. Dipteroc. Schizomyia galiorum Kieff. Blüthen verdickt und geschlossen bleibend. Bitsch.

Genista tinctoria L. Dipteroc. Contarinia n. sp. Haselnussgrosse, fleischige, vielkammerige Stengelschwellung. V. i. E. Metz. Diese Deformation wurde früher einer Cynipide (Aulax genistar) zugeschrieben; Hieronymus fand in denselben fleischrothe Cecidomyidenlarven. Die von mir beobachteten Exemplare, welche von Herrı Abbé Friren gesammelt wurden, enthielten gelbliche Springmaden.

Hieracium boreale Fr. Dipteroc. Cystiphora hieracii (F. Lw.) Roth gefärbte, kreisförmige Blattparenchymgallen. Legit. Frir en. Metz.

Holcus. 1. Dipteroc. Mayetiola holci Kieff. Sattelförmige Verdickung unter den Blattscheiden, meist an den unteren Knoten. Auf H. lanatus L. und mollis L. Larven weiss, zu mehreren; Lebensweise wie bei Mayetiola dactylidis. Waldrand bei La Grange und Bitsch. Whitehead hat auf Holcus lanatus L. ein Tönnchen beobachtet, welches er für das Puparium von May. destructor hielt; eine Deformation wurde nicht angegeben. (Whitehead et Gray. Report of the Commissioners appointed by the Governement to enquire into the present visitation of the Hessian fly on corn crops in Great Britain. London 1887, p. 7.)

*2. Phytopt. Vergrünung der Aehrchen mit Bildung neuer gestielter Aehrchen, welche aus den ersten hervorragen; Spelzen verdreht. Auf H. lanatus. Bitsch.

*Hypericum hirsutum L., humifusum L. und montanum L. Dipteroc. 1. Diplosis Giardianu Kieff. ${ }^{1}$ ) Die von Géné auf H. perforatum beobachtete, einer Frucht ähnliche, kuglige, schön roth gefärbte, aus zwei schalenförmigen, sich mit den Rändern berührenden Blättern bestehende Deformation fand ich häufig auf beiden genannten Arten im Walde von La Grange. Wie Géné und Giard angeben, findet die Metamorphose in der Galle statt; die Nymphenhaut bleibt zwischen den schalenförmigen Blättern zurück. Es mag wohl hier der Fall vorhanden sein, den ich für Rhopalomyia foliorum H. Lw. beobachtet habe; diese Mücke hat mehrere Generationen in demselben Jahre; bei der ersten findet die Verwandlung in dem Cecidium statt; bei der letzten dagegen verlassen die Larven ihren Aufenthaltsort im October und November, um in der Erde zu überwintern. Ebenso habe ich früher für die Hypericum-Mücke die

1) Bei der Beschreibung wurde aus Versehen Diplosis Giardi geschrieben. 
Beobachtung gemacht, dass die Larven, welche im Spätherbst in der Galle vorhanden waren, sich zur Metamorphose in die Erde begaben.

2. Dasyneura serotina Winn. Diese um Bitsch auf H. perforatum und pulchrum häufige Deformation wurde auch anf H. hirsutum und montanum bei La Grange, und H. humifusum und quadrangulum bei Bitsch beobachtet.

Lathyrus odoratus L. Dipteroc. Dasyneura sp. Blüthenschwellung. Larven roth, in Mehrzahl. Gentringen.

Lathyrus pratensis L. Dipteroc. Auf dieser Pflanze wurden bisher in Lothringen drei Missbildungen beobachtet, nämlich 1. eine Triebspitzendeformation: Dasyneura sp.?; 2. eine Blattrandrollung nach oben bis zur Mittelrippe, nicht oder kaum verfärbt und kaum merklich verdickt: Dasyneura sp.?; 3. eine kaum verdickte Blättchenfaltung: Dasyneura sp.? Diesen habe ich eine vierte und fünfte hinzuzufügen: erstere war, mit der Blattrandrollung, häufig im Walde von La Grange zu sehen; an den Trieben und in den Blattachseln zeigen sich die Nebenblätter beiderseits eingerollt, überaus stark verdickt, knorpelig, gelb bis roth gefärbt; der Erzeuger ist Macrodiplosis Bellevoyei Kieff., dessen Verwandlung in der Erde stattfindet. 4. Blüthenschwellung. Contarinia sp. Gentringen.

Lathyrus silvestris L. Dipteroc. 1. Dasyneura sp.? Blüthen geschlossen bleibend und stark geschwollen. V. i. E. Wald von Gentringen.

2. Contarinia silvestris Kieff. ${ }^{1}$ ) Hülsen mit kaum merklichen blassen Beulen. V. i. E. Mit voriger.

Lythrum hyssopifolium L. $\left.{ }^{2}\right)$ Coleopteroc. Nanophyes hemisphaericus 01. Rundliche oder längliche, erbsen- bis schlehendicke Stengelschwellung. Dieselbe kommt häufig an der Basis (erste Gene-

1) Vgl. "Meine Antwort an den Herrn Zeichenlehrer Rübsa amen und an den Herm Docenten Dr. F. Karseh, nebst Beschreibung neuer Gallmücken, 1897, S. 15.

2) Auf dieser Pflanze lebte auch Myzus lythri Schr. Herr Dr. Horvath war so freundlich, mir diese Art und eine Anzahl anderer Aphiden zu bestimmen. Ich habe früher Herm Rübsaam en, auf seine Bitte, über 170 Aphiden- und Cocciden-Arten zukommen lassen. Nach jeder Sendung wurde mir mitgetheilt, dass dieselbe in gutem Zustande angekommen sei und in einem vom 23. Januar 1895 datirten Schreiben wurde mir die Erfüllung eines längst gegebenen, bis hente aber noch nicht gehaltenen Wortes mit folgenden Ausdrücken angekündigt: "Ich denke, dass ich im Februar dazu komme, Ihre Aphiden alle zu bestimmen." In der Oeffentlichkeit äusserte sich dagegen Herr R. (1896) auf folgende Weise: "Alle diese Aphiden sind so schlecht conservirt und in einem so traurigen Zustande angekommen, dass eine Ueberführung ins Berliner zoologische Museum zur Unmöglichkeit wurde." 
ration?), seltener in der Inflorescenz (zweite Generation? oder verspätete Exemplare?) vor und zeigt gewöhnlich nur eine Larvenkammer. Ich beobachtete diese Cecidien am 21. September in grosser Anzahl auf feuchten Aeckern, zwischen Garsch und Monhofen, in der Nähe der Mosel. Aus den Inflorescenz-Gallen kamen in den folgenden Tagen mehrere Käfer zum Vorschein, während die Schwellungen des Stengelgrundes sämmtlich unbewohnt und mit einem Flugloche versehen waren. Diese Deformation wurde zuerst von Perris (1854) bekannt gemacht. Kaltenbach (Die Pflanzenfeinde) hat nach Dufour Nanophyes lythri als Erzeuger gehalten, während Perris richtig Nanophyes hemisphaericus angegeben hatte. Dieselbe scheint wenig verbreitet zu sein. Nach der "Faune de la Lorraine" von Godron soll Nanophyes hemisphaericus häufig auf Lythrum Salicaria bei Metz, an der Mosel, zu finden sein; es wird aber wohl auch hier eine Verwechselung vorliegen, da der betreffende Käfer nur auf Lythrum hyssopifolium lebt, diese Pflanze aber in Lothringen zu den seltenen gehört. In Deutschland wurde die Deformation bisher nicht beobachtet. Szepligeti erwähnt derselben für Ungarn.

Medicago falcata L. Dipteroc. Contarinia medicaginis Kieff. Blüthen geschlossen bleibend und verdickt. Gehnkirchen.

Pinus silvestris L. Coleopteroc. Brachonyx indigena Hbst. Deformation der Nadeln. Die beiden Nadeln einer Scheide bleiben dicht aneinander gedrückt, erreichen nur die Hälfte oder ein Drittel der normalen Grösse, zeigen sich in der Mitte stark verdickt und stellen so ein spindelförmiges Cecidium dar. In einem inneren Hohlraum lebt die Larve einzeln und erleidet ihre Verwandlung an derselben Stelle. Der Käfer erscheint im Juli, nachdem er sich zuvor eine kreisrunde Oeffnung in die Gallenwand genagt hat. Bitsch.

Poa nemoralis L. 1. Dipteroc. Mayetiola Joannisi Kieff. Kaum merkliche Stengelschwellung an den unteren Halmknoten. Verwandlung der Larve wie bei May. destructor. Wald von La Grange.

2. Helminth. Tylenchus sp ? Blutrothe, meist spindelförmige Schwellungen an Blättern. An derselben Stelle wie vorige Deformation.

Polygonum amphibium L. Die von mir in den Berliner Ent. Nachr. (1895) beschriebene Triebspitzendeformation wird durch Aphalara maculipennis Fr. Lw. hervorgerufen; mit dieser Art lebte auch Aphalara exilis. Die Bestimmung verdanke ich Herrn Dr. Horvath. Unter den Aphalara-Nymphen lebten MycodiplosisLarven; eine Pilzbildung war aber nicht vorhanden. Dieselben Pflanzen waren auch durch Dasyneura polygoni deformirt; mehrere 
Larven dieser Mücken-Art lagen tod in der Blattrollung und trugen als Parasiten eine blutrothe Lestodiplosis-Art.

Populus alba L. Dipteroc. Harmandia sp.? Erbsengrosse, kuglige, dickwandige, das Blatt durchwachsende Gallen; auf der Blattoberseite, wo sie kaum hervorragen, öffnen sie sich spaltförmig. Wald von La Grange.

Populus Tremula L. Lepidopt. Nepticula apicella St. Kaum merkliche Schwellung der Blattstielspitze. Häufig im Walde von La Grange.

Dipteroc. 1. Agromyza Schineri Gir. Einseitige Rindenschwellung in der Nähe der Zweigspitze.

*2. Contarinia sp. Enge, fleischig verdickte, glatte, glänzende Blattrandrollung nach oben. Mit voriger, um Bitsch.

Phytopt. Pliytoptus diversipunctatus Nal. Anschwellung der Blattdrüsen am Grunde des Blattes zu rothen unregelmässig gestalteten Gallen. Mit voriger.

Quercus. Hymenopt. 1. Andricus Lambertoni Kieff. Knospengalle: holziğ, längsgefurcht, 5-6 $\mathrm{mm}$. hoch, unterseits kegelförmig und $3 \mathrm{~mm}$. dick, oberseits in eine hohle, hornförmige Verlängerung auslaufend; letztere $1^{1 / 2}$ mal so lang als der kegelförmige Theil. Auf Q. pedunculata oder sessiliflora, bei Bitsch.

2. Neuroterus aprilinus Schl. Knospen stark angeschwollen, $8 \mathrm{~mm}$. lang und $3 \mathrm{~mm}$. breit, mit einem oder zwei grossen Innenräumen. Q. sessiliflara. Mai. Bitsch.

*Dipteroc. Knospen seicht geschwollen und verlängert, später absterbend. Mit rothen Gallmückenlarven. Auf Q. sessiliflora. Bitsch.

Hemipt. Asterodiaspis quercicola Bché. Vertiefungen und Umwallungen auf den Zweigen von $Q$. pedunculat a und sessiliflora hervorrufend. Häufig um Bitsch.

Ribes grossularia L. Dipteroc. Contarinia n. sp. Blüthen geschlossen bleibend und verdickt. V. i. F. Zwischen Sierck und Montenach.

Salix aurita, Caprea und cinerea L. Dipteroc. 1. Bertieria nervorum Kieff. Kaum merkliche Schwellung der Mittelrippe oder des Stieles der Blätter von S. Caprea. Die Larve, welche im Spätherbst untersucht wurde, zeigte dieselbe (iräte wie die auf S. a urita lebende $B$. nervorum, jedoch war sie nicht ron gelber, sondern von weisser Farbe.

2. Bertieria rosariella Kieff. Knospendeformation. Das Cecidium stellt eine sehr kleine Rosette dar, welche in ihrer Bildung den bekannten durch Cecid. rosaria erzeugten Weidenrosen ähnlich 
ist; durch seine winzige Gestalt ist es aber von letzteren unschwer zu unterscheiden, da es nicht einmal die Grösse einer Erbse erreicht; auch scheint es nur in den Blattachseln vorzukommen, während die grossen Weidenrosen von mir stets an den Zweigspitzen beobachtet wurden. Larve ohne Gräte. Auf S. aurita und cinerea. Bitsch.

*3. Bertieria superna Kieff. Knospendeformation. Knospe nicht grösser als die normalen, aber von spitz-eiförmiger Gestalt und aus trockenen bräunlichen Schuppen zusammengesetzt; Blattpolster nicht angeschwollen. Die Larve, welche ich ebenso wie die von Cec. rosariella im März untersucht habe, zeigt eine Gräte, die nur aus zwei hyalinen, am Grunde nicht zusammenhängenden Spitzen besteht. Ich fand solche um Bitsch auf S. aurita, Caprea und cinerea.

*4. Bertieria gemmicola Kieff. Knospendeformation. Knospe grün und von den normalen nur dadurch verschieden, dass sie stark verdickt und daher von den durch Cryptocampus saliceti Fall. bewirkten Schwellungen nicht zu unterscheiden ist. Die Larve steht nicht, wie die der beiden vorigen Arten, der $\boldsymbol{B}$. rosaria, sondern der $B$. dubia nahe; ihre Gräte ist gestielt und dunkelbraun. Auf S. a urita und cinerea, bei Bitsch

Silene nutans I. Lepidopt. Spindelförmige Stengelschwellung. Raupe einzeln. In einem Garten in Luxemburg.

Sisymbrium Sophia L. Dipteroc. Contarinia ruderalis Kieff. Schwammige, weiss gefärbte Inflorescenz-Gallen. V. i. E. Bitsch.

Sonchus oleraceus L. Dipteroc. Contarinia sonchi Kieff. Blüthenköpfe geschlossen und schwach verdickt. Die Mücken unterscheiden sich von $C$. Schlechtendali Rübs. durch die Flügel, welche mit Ausnahme der Basis schwärzlich erscheinen, sowie durch das erste Tarsenglied, das stets schwarz ist. Schloss La Grange.

Triticum vulgare L Dipteroc. Mayetiola destructor Say. Kaım merkliche Schwellung an den unteren Halmknoten. Im September bei Diedenhofen beobachtet.

T'rifolium medium L. Dipteroc. Dasyneura axillaris Kieff. Blattachselgallen, ähnlich den von Dasyn. ignorata Wachtl auf Medicago sativa bewirkten. Missbildungen. V. i. E. Wald von La Grange. 


\section{$2 \mathrm{BHL}$ Biodiversity Heritage Library}

Kieffer, J.-J. 1897. "Nachtrag zu den Zoocecidien Lothringens." Berliner entomologische Zeitschrift / herausgegeben von dem Entomologischen Verein in Berlin 42, 17-24. https://doi.org/10.1002/mmnd.18970420107.

View This Item Online: https://www.biodiversitylibrary.org/item/101307

DOI: https://doi.org/10.1002/mmnd.18970420107

Permalink: https://www.biodiversitylibrary.org/partpdf/82133

\section{Holding Institution}

Harvard University, Museum of Comparative Zoology, Ernst Mayr Library

\section{Sponsored by}

Harvard University, Museum of Comparative Zoology, Ernst Mayr Library

\section{Copyright \& Reuse}

Copyright Status: Public domain. The BHL considers that this work is no longer under copyright protection.

This document was created from content at the Biodiversity Heritage Library, the world's largest open access digital library for biodiversity literature and archives. Visit BHL at https://www.biodiversitylibrary.org. 